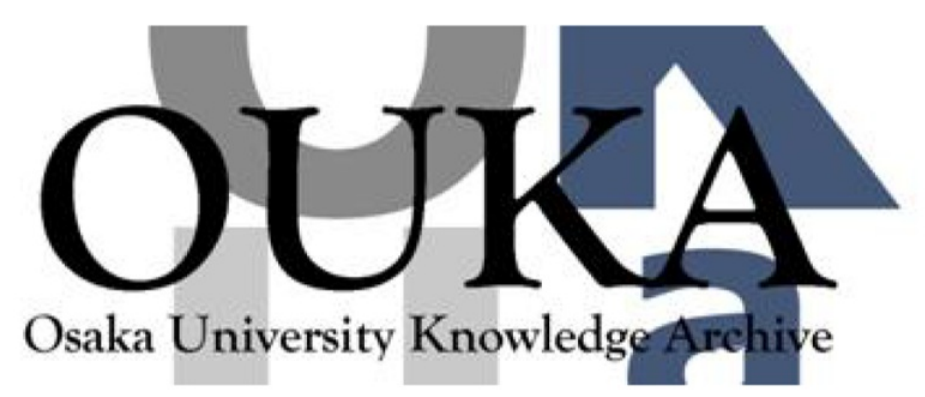

\begin{tabular}{|c|l|}
\hline Title & $\begin{array}{l}\text { A Hierarchical Image Transmission System for } \\
\text { Multimedia Mobi le Communication }\end{array}$ \\
\hline Author(s) & $\begin{array}{l}\text { Morimoto, Masakazu; Okada, Minoru; Komaki, } \\
\text { Shozo }\end{array}$ \\
\hline Citation & $\begin{array}{l}\text { IEICE Transactions on Communications. E80-B(5) } \\
\text { p. 779-p. 781 }\end{array}$ \\
\hline Issue Date & $1997-05$ \\
\hline oaire:version & VoR \\
\hline URL & https://hdl. handle. net/11094/3322 \\
\hline rights & copyright $\odot 2008$ IEICE \\
\hline Note & \\
\hline
\end{tabular}

Osaka University Knowledge Archive : OUKA

https://ir. Library. osaka-u. ac. jp/

Osaka University 


\title{
LETTER \\ A Hierarchical Image Transmission System for Multimedia Mobile Communication
}

\author{
Masakazu MORIMOTO ${ }^{\dagger}$, Student Member, Minoru OKADA ${ }^{\dagger}$, and Shozo KOMAKI ${ }^{\dagger}$, Members
}

SUMMARY This paper optimizes a hierarchical image transmission system based on the hierarchical modulation scheme in a band-limited Rayleigh fading channel. Authors analyze relations between hierarchical parameters and the image quality, and show that the existence of optimum hierarchical parameter that maximizes the received image quality.

key words: hierarchical modulation, multipath fading, multimedia mobile communication

\section{Introduction}

Recently, there has been an increasing demand for multimedia transmission, such as transmission of text, data, voice and images, over mobile communication systems. Multimedia transmission requires highly reliable and high speed digital transmission. However, in mobile communication systems, since multipath fading degrades the transmission reliability and the frequency bandwidth is strictly limited, it is difficult to realize the multimedia transmission without applying techniques which overcome these problems.

To overcome these problems, hierarchical transmission system has widely been studied. Hierarchical transmission system, composed of hierarchical source coder and corresponding channel coder, divides the information into several layers according to their significance, and transmits each layer with different reliability according to the layers.

As a hierarchical channel coder, unequal error protection coding method has been studied [1], [2]. Hierarchical modulation method [3], [4] is another way to realize the hierarchical transmission. Ramchandran [3] proposed a hierarchical modulation based image transmission system for terrestrial HDTV (High Definition Television) broadcasting, and investigate the system performance in terms of both coverage area and subjective quality.

In a previous paper [4], we have proposed an optimization method to maximize the spectrum efficiency of the hierarchical modulation system for satellite broadcasting and shown that the proposed system can improve the spectral efficiency performance. However, previous study analyzes only the spectrum efficiency performance and there is no study about maximization of image quality performance.

\footnotetext{
Manuscript received February 7, 1997.

${ }^{\dagger}$ The authors are with the Faculty of Engineering, Osaka University, Suita-shi, 565 Japan.
}

In this paper, we propose an optimum hierarchical image transmission system based on hierarchical modulation scheme to maximize the transmitted image SNR performance. In this system, we use a hierarchical QAM (quadrature amplitude modulation) scheme to give unequal transmission reliability depending on the layers of ADCT (adaptive discrete cosine transform, [5])-based compressed images. The signal constellation of the hierarchical QAM used for the proposed system is optimized to maximize received image quality.

\section{System Model}

In order to realize a two layers modulation scheme we use a hierarchical 16 QAM modulator. Figure 1 shows a constellation diagram of the hierarchical 16QAM signal, where $D_{1}$ and $D_{2}$ are the minimum distance between clusters and the minimum distance within the cluster, respectively. In this figure, the base information bits correspond to the 4 clusters, and the refinement information bits decide one of the 4 constellation points within each cluster. Since $D_{1}$ is larger than $D_{2}$, the base information layer will be transmitted with lower bit error rate than the refinement information layer.

Let $\lambda=D_{2} / D_{1}$ be the modulation parameter of the hierarchical 16QAM, which represents the reliability between two layers. When $\lambda$ approaches to zero, the reliability of the base information is improved and the refinement layer drops it's reliability, and if $\lambda=0$, the signal constellation becomes a conventional QPSK

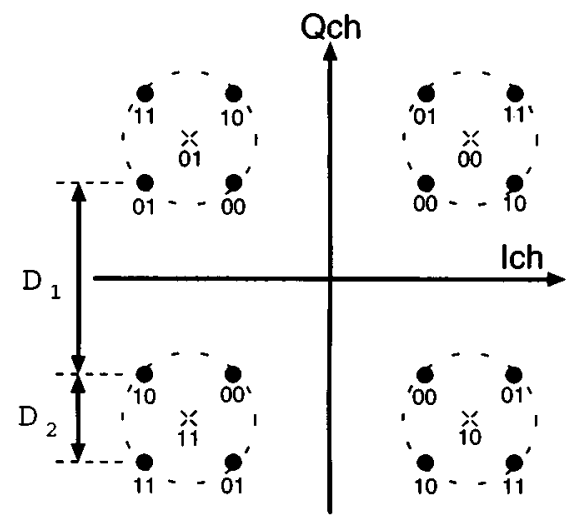

Fig. 1 Constellation diagram of the hierarchical 16QAM. 


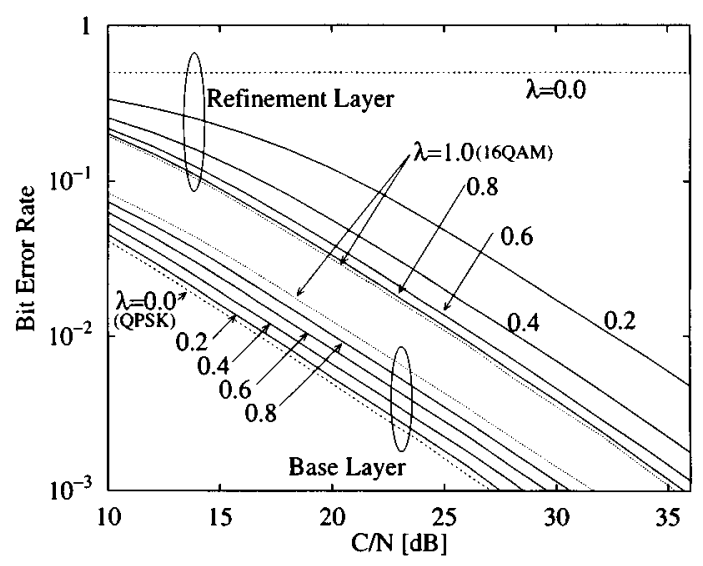

Fig. 2 BER performance of hierarchical 16 QAM against average CNR of a Rayleigh fading channel.

(quadrature phase shift keying) and only base layer is transmitted. On the other hand, if $\lambda=1$, the signal constellation becomes a conventional rectangular 16 QAM and each layer has equal reliability. By controlling $\lambda$, we can set bit error rate (BER) of the base and refinement layers, $P_{e 1}$ and $P_{e 2}$, respectively. They are approximately given by

$$
\begin{aligned}
P_{e 1}(\lambda) & =\frac{1}{4} \operatorname{erfc}\left(\sqrt{\frac{\gamma}{4 \lambda^{2}+4 \lambda+2}}\right) \\
& +\frac{1}{4} \operatorname{erfc}\left(\sqrt{\gamma \cdot \frac{4 \lambda^{2}+4 \lambda+1}{4 \lambda^{2}+4 \lambda+2}}\right)
\end{aligned}
$$

and

$$
P_{e 2}(\lambda)=\frac{1}{2} \operatorname{erfc}\left(\sqrt{\frac{\lambda^{2} \gamma}{4 \lambda^{2}+4 \lambda+2}}\right),
$$

where $\gamma$ is the CNR at the receiver front end.

Figure 2 shows a BER performance of the hierarchical 16QAM in a Rayleigh fading channel. This figure shows that the hierarchical modulator improves the BER of base layer transmission at the sacrifice of refinement layer's BER.

\section{System Optimization}

In a fading channel, CNR varies dynamically. When we apply conventional, non-hierarchical transmission system to fading channel, transmission error frequently occur. But, in this case, there can be high-bit rate transmission when the channel conditions are good. On the other hand, when we apply the proposed hierarchical transmission system to the fading channel, we can achieve the high-reliability transmission for the base layer information. In this case, the error occurs more frequently to the refinement layer's transmission, but the total received quality is improved because the refinement information is less significant and insensitive to the transmission error.
Table 1 Simulation parameters.

\begin{tabular}{|c|c|c|}
\hline Image Size & \multicolumn{2}{|c|}{$256 \times 256,8 \mathrm{bit} / \mathrm{pixel}$} \\
\hline Compression Rate & $12.5 \%(1 / 8,1 \mathrm{bit} / \mathrm{pixel})$ \\
\hline Simulated Image & "GIRL" & "AERIAL" \\
\hline Compressed Image SNR & $35.2 \mathrm{~dB}$ & $28.5 \mathrm{~dB}$ \\
\hline Average MSE (Layer 1) & 290.7 & 538.7 \\
\hline Average MSE (Layer2) & 20.7 & 54.2 \\
\hline Fd·T & \multicolumn{2}{|c|}{0.004} \\
\hline Pilot Symbol Duration & \multicolumn{2}{|c|}{16} \\
\hline
\end{tabular}

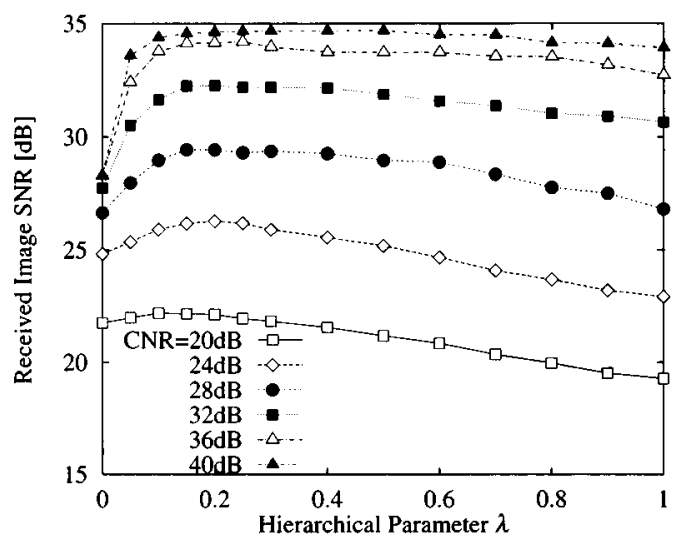

(a) GIRL

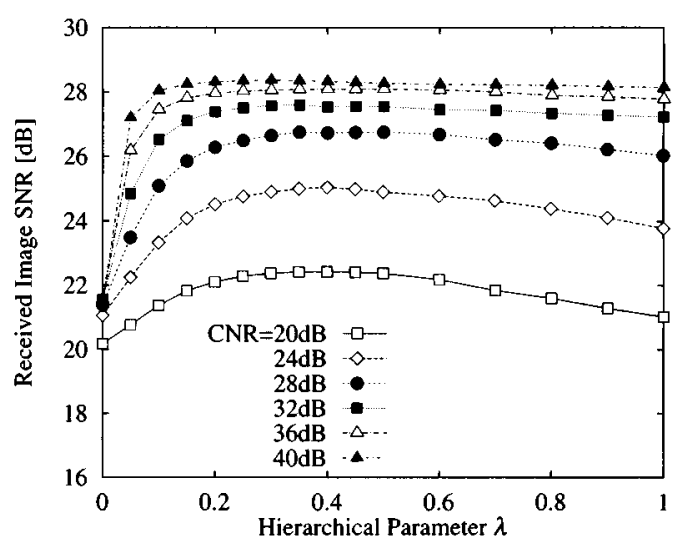

(b) AERIAL

Fig. 3 Received image SNR against the modulation parameter $\lambda$.

In this section, we consider an optimization method of the proposed hierarchical transmission system. When we set the hierarchical modulation parameter, $\lambda$, to small value, we can transmit the base layer with high reliability. But, in this case, the refinement layer becomes difficult to receive. On the other hand, when we set $\lambda$ to large value, we can improve the refinement layer reliability. But it makes worse the base layer's reliability. If we can know the statistical channel characteristic and receiving performances from each layer, we can optimize the $\lambda$ to maximize the received image SNR performance.

In this paper, we investigate the transmitted image SNR performance of the proposed hierarchical system 
in a slow flat Rayleigh fading environment. Here, we apply the pilot symbol assisted modulation (PSAM) to estimate the attenuation and phase shift due to fading. Simulation parameters are shown in Table 1.

In this simulation, we compress the image information by ADCT-based image coding scheme and divide the image data into two layers according to the layering algorithm [6] of DCT coefficients. Here, we use the two test images which are refereed as "GIRL" and "AERIAL." Image compression rate and each layer's average mean square error (MSE) which caused by one bit transmission error are also shown in Table 1.

Figure 3 show the received image SNR performance against the hierarchical modulation parameter, $\lambda$. Form these figures, the received image SNR performance can be maximized at $\lambda=0.2$ and $\lambda=0.4$ for

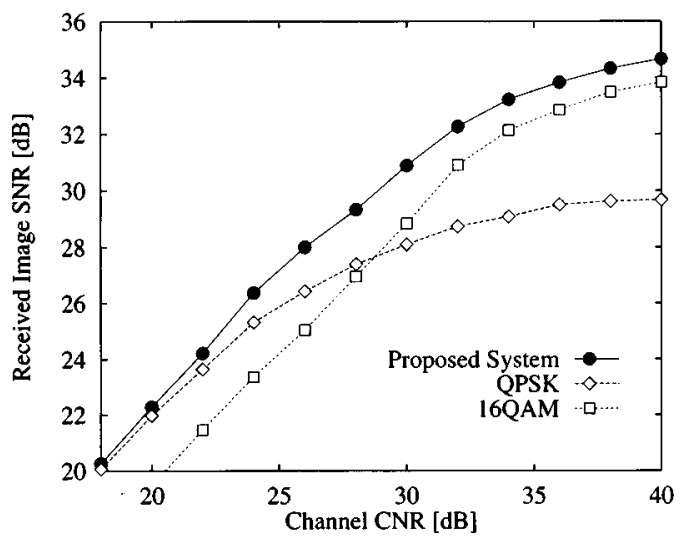

(a) GIRL

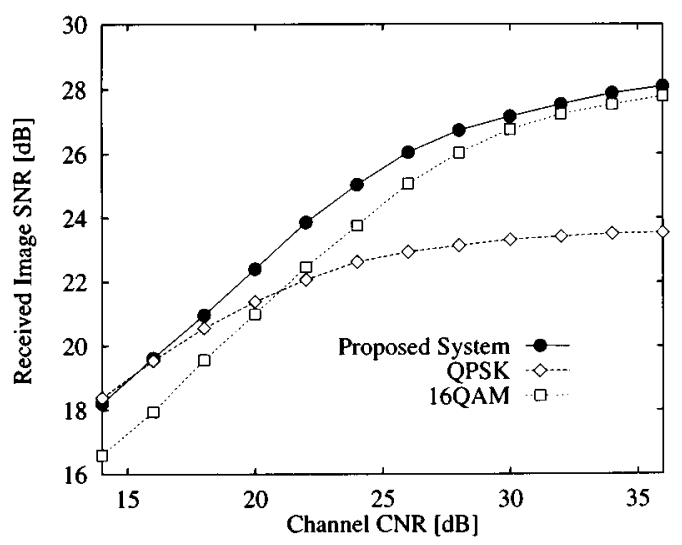

(b) AERIAL
"GIRL" and "AERIAL" respectively. Since the refinement layer of "AERIAL" is more sensitively to transmission error, the optimum value of $\lambda$ becomes larger.

\section{Simulation Results}

Figure 4 show the SNR performance against the average channel CNR. In Fig. 4, the optimized hierarchical system gives better SNR performance than both QPSK and 16QAM systems when CNR is greater than $18 \mathrm{~dB}$ and can achieve comparable performance to QPSK when CNR is less than $18 \mathrm{~dB}$.

From these results, it is clear that the proposed system can improve the reconstructed image SNR performance effectively in the Rayleigh fading channel.

\section{Conclusion}

In this paper, we have analyzed the relations between the hierarchical parameter and the image quality, and shown the optimum hierarchical parameter in the Rayleigh fading channel which maximizes the received image quality. We have also clarified that the proposed system can improve the received image performance in a fading channel.

\section{References}

[1] N. Matoba and S. Yoshida, "Still image transmission using unequal error protection coding in mobile radio channel," IEICE Trans., vol.J78-B-II, no.3, pp.93-101, March 1995.

[2] N. Yamane, Y. Morikawa, H. Sawai, H. Hamada, and K. Hatada, "Methods of rate-allocation to bit-planes in combined source-channel coding," IEICE Trans., vol.J74-B-I, no.1, pp.74-81, Jan. 1991

[3] K. Ramchandran, A. Ortega, K.M. Uz, and M. Vetterli, "Multiresolution broadcast for digital HDTV using joint source/channel coding," IEEE J.Commun., vol.11, no.3, pp.6-23, Jan. 1993.

[4] M. Morimoto, H. Harada, M. Okada, and S. Komaki, "A study on power assignment of hierarchical modulation schemes for digital broadcasting," IEICE Trans., vol.E77-B, no.12, pp.1495-1500, Dec. 1994.

[5] W.H. Chen and C.H. Smith, "Adaptive coding of monochrome and color images," IEEE Trans. Commun., vol.COM-25, pp.1285-1292, Nov. 1977.

[6] K. Manabe, T. Tanaka, and S. Ohtsuka, "A variable bit rate video codec for ATM networks and its robustness against cell losses," IEICE Trans., vol.J76-B-I, no.1, pp.40-47, Jan. 1993.

Fig. 4 Received image SNR against the channel average CNR. 